\title{
Optimum Design of Brake Pedal for Trucks Using Structural Optimization and Design of Experiment Techniques
}

\author{
Oğuz Doğan ${ }^{*}$, Onur Can Kalay², Emirhan Kartal² and Fatih Karpat ${ }^{2}$ \\ 0000-0003-4203-8237, 0000-0001-8643-6910, 0000-0002-4259-8341, 0000-0001-8474-7328 \\ 1 Department of Mechanical Engineering, Faculty of Engineering and Architecture, Kahramanmaras Sutcu Imam University, \\ ${ }^{2}$ Department of Mechanical Engineering, Faculty of Engineering, Bursa Uludag University, Bursa, 16059, Turkey
} Kahramanmaras, 46040, Turkey

\begin{abstract}
Along with the recent developments and innovations in the automotive and heavy vehicle industry, the performance requirements such as fuel efficiency, emissions reduction, low manufacturing cost, etc., keep increasing day by day. In order to have a place in the world market, it is essential to meet these requirements. Along with the other strategies, vehicle weight reduction is one of the most critical strategies in the heavy vehicle industry. This paper aims to obtain an optimal design of a truck brake pedal by employing topology and shape optimization. To accomplish this goal, the material used for an existing brake pedal is unchanged as this study focuses on reducing the weight of the existing brake pedal without material substitution. The rough dimensions of the brake pedal are designed by inspiring the results of the topology optimization. To determine the precise dimensions of the brake pedal design of experiment (DOE) and shape optimization studies are conducted respectively. Wall and flange thickness are defined as variable parameters for the DOE. Three different values are selected for each design parameter. Stress analyses are conducted by using the finite element method for nine cases. As a result of the studies mentioned so far, two responses are obtained. In order to obtain minimum weight value, which is possible shape optimization is performed by using fmincon function in $\mathrm{MATLAB}^{\circledR}$. As a result of the study, the mass reduction of the brake pedal is \%50, and it is shown that the developed method can be used to design a lightweight truck brake pedal.

Keywords: Design of experiment; Finite element method; Light weighting; Structural optimization
\end{abstract}

\author{
* Corresponding author \\ Oğuz Doğan \\ oguzdogan@ksu.edu.tr \\ Address: Department of Mechanical \\ Engineering, Faculty of Engineering \\ and Architecture, Kahramanmaras \\ Sutcu Imam University, \\ Kahramanmaras, 46040, Turkey
}

Tel: +903443004587

Research Article

$\begin{array}{ll}\text { Manuscript } & \\ \text { Received } & 21.08 .2020 \\ \text { Revised } & 10.10 .2020 \\ \text { Accepted } & 12.10 .2020\end{array}$

Doi: 10.30939/ijastech..783552

\section{Introduction}

Today, optimizing fuel efficiency, carbon dioxide emission, and manufacturing costs in the automotive and heavy vehicle industry emerge as one of the most significant tasks for designers with developing technology. In this aspect, a mass reduction starting from the smallest component of the vehicle can make a great contribution to this aim.

The cost of the power transmission components of a tractor is approximately $25-30 \%$ of its total cost [1]. A cost reduction to be realized return to companies as profitability in mass production. Thus, studies on obtaining optimized lightweight components in the automotive and heavy vehicle industry to decrease manufacturing cost and boost efficiency, are increasing day by day. The energy to be consumed by the vehicle can be decreased by reducing the vehicle mass with various optimization methods, and optimized designs with a longer life can be obtained.

The researchers persuaded the objective of minimizing the weight of the vehicle components such as brake pedals, compressor brackets, and chassis, etc. considering the safety aspects in the literature. Thus, structural optimization and design of experiments studies were considerably useful and frequently preferred instruments in order to achieve this goal. The structural optimization methods include topology, shape, and size optimizations. Thus, topology optimization plays an important role in the initial design phase for conceptual design. However, topology optimization provides a load-balanced material distribution for the initial design phase, and the obtained dimensions regarding topology optimization were not precise. Thus, shape and size optimization methods were widely used to complete the final design. 


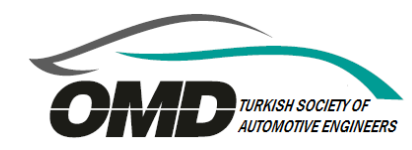

Design of experiment (DOE) is also a functional method to determine the optimum dimensions, and more easily applied to develop optimum designs regarding improvements in the computer-aided engineering (CAE) tools. There are many studies applying the optimization methods to weight reduction of the vehicle components to find an optimum design in the literature.

Dogan et al. analyzed the Power take-off (PTO) finger, which currently used in the market and proposed a new design of PTO finger for the tractor clutch. For this aim, the study employed topology and size optimization methods. The boundary conditions were defined, and the stress-life based fatigue analysis was conducted via finite element analysis (FEA) to determine the crack initiation points on the PTO finger. The finger height and thickness were determined as the design variables. Thus, the researchers performed a response surface method (RSM) to obtained objective functions due to the complexity of describing the functions analytically. Thus, the optimum setting of the experimental factors was determined. The study revealed a method to find optimum PTO finger design in order to maximize fatigue life while minimizing the von - Mises stress, deformation, and the mass. As a result, a stiffer and lightweight design of the PTO finger was obtained by comparing a PTO finger used in the market [1]. Karpat et al. overdesigned a chain link to optimize stress and mass values. First, the equivalent von Mises distribution of the chain-link was determined via the finite element method. Thus, it was obtained that the highest von - Mises stresses were observed in the top radius region of the chain link. The safety factor of the chain-link was also calculated in the study. It was observed that the current design was too safe for a static system. Thus, a shape optimization method was applied to optimize the mass of the chain link. The chain inner radius and hole radius values were determined as the design variables. The stress and mass equations were determined using curve fitting. The obtained results were compared with the current chain link design. Finally, the mass of the chain-link was reduced by nearly $50 \%$ by using RSM and shape optimization methods [2]. Dogan et al. studied on the optimizing the truck clutch PTO finger. The finite element analysis, topology, and shape optimization methods were used instead of the cost and time consumed experimental tests in the study. Two design variables were defined, and the RSM of the maximum von - Mises stress, bending displacement, and the mass was derived. Thus, the $25.3 \%$ and $27.9 \%$ reduction in von - Mises stress and bending displacement was obtained, while, a $53.2 \%$ improvement in fatigue life was achieved in the new design of the truck clutch PTO finger [3].

Ergenc et al. reduced the weight of the automotive brake pedals and validated the new design by utilizing a single pneumatic pedal test system. The fatigue life of the five different automotive brake pedal was determined via the finite element analysis, and the stress-life method was used. As a result, a $12 \%$ mass reduction of the brake pedal was achieved
[4]. Kaya et al. proposed a new design for the failed clutch fork via RSM. The researchers, first, analyzed the current clutch fork design in case of stress-life based fatigue performance. The shape and topology optimization methods were employed for the new design of the clutch fork. The rigidity was improved by nearly $37 \%$ since a $9 \%$ and $24 \%$ reduction in the mass, and the stress was obtained with the optimized design in comparison to the original clutch fork [5]. Cavazzuti et al. presented a topology optimization-based method for the automotive chassis design. Five different loading conditions were considered to define the boundary conditions. The study aimed the weight reduction via optimization methods such as topometry and size optimization methods to reached optimum design of the automotive chassis. As a result, a considerably noticed weight reduction was obtained in comparison to the current chassis design [6]. Kurkure and Sadaphale studied the optimization of the brake pedal used in the cars in order to weight reduction via the topology optimization method. For this aim, the finite element method was used in the study. As a result, an $18.29 \%$ reduction in the brake pedal weight was obtained [7]. Ebrahimi and Behdinan proposed a new approach to design cross-car beam assemblies via structural optimization methods. Thus, size and shape optimization methods were also used since the obtained dimensions after the topology optimization was not precise. The design criteria and the objective function were defined for the conceptual process. Thus, the detailed design process was achieved based on structural optimization methods. The weight of the cross-car beam assemblies was reduced to $5.1 \mathrm{~kg}$ from the $8.17 \mathrm{~kg}$ with the proposed approach [8]. Chang and Lee redesigned a compressor bracket with the topology optimization, and the model was verified with an experimental test setup under the static and dynamic loading conditions. The objective function was defined to minimize the combined compliance. The new compressor bracket design was compared with the current design in order to assess the natural frequency and mass values. As a result, a $31 \%$ mass reduction was observed in the study [9]. Sudin et al. proposed a new design for the automotive brake pedal. The researchers used topology optimization to reduce weight. The design space was defined, and the topology optimization analysis was converged after 45 iterations. The optimized brake pedal design was compared with the initial design against the mass, deformation, von - Mises stress, and the safety factor. A $22 \%$ weight reduction was observed with the optimized brake pedal design [10].

The researchers also applied different optimization techniques in order to achieve the optimum design in the literature. Dhande et al. focused on the conceptual design of the brake pedal with using the variable-material since the brake pedals mostly made of metal. Three different materials and the five different sections of the brake pedal arm profile were analyzed via the finite element method against deformation, stress, and the mass. It was obtained that the composite material was more beneficial to decrease brake pedal weight in 
comparison with the steel and aluminum materials [11]. Romero and Queipo formulated and solved the reliabilitybased design optimization (RBDO) and deterministic design optimization (DDO) models for the FSAE brake pedal via risk allocation analysis. The RBDO and DDO models were solved for the stress and buckling modes. As a result, it was observed that the models solved with the RBDO were lightweight than the models solved with the DDO [12].

In this study, it was aimed to achieve an optimum design for the truck brake pedal by using topology and shape optimization methods. The material used for an existing brake pedal was unchanged, and the optimization methods applied without material substitution. The wall and flange thickness values were defined as the design parameters for DOE. Thus, three different values from each design parameters were selected, and two equations regarding mass and stress were derived via the curve-fitting function of MATLAB ${ }^{\circledR}$ (MathWorks Inc., Natick, USA). The "fmincon" function was employed to minimize the weight of the truck brake pedal, and shape optimization analysis was conducted to found the precise dimensions of the optimized brake pedal design. The finite element analysis was performed via ANSYS ${ }^{\circledR}$ Workbench (ANSYS Inc., Canonsburg, USA) to confirm the durability of the redesigned brake pedal model. A lightweight final design of the truck brake pedal was obtained with the proposed model.

\section{Methodology}

\subsection{First design and finite element stress analysis of current brake pedal}

In this study, a design procedure is described for brake pedal for heavy trucks by using structural optimization and design of experiment method. The rough design of the brake pedal is defined in accordance with the design space, which is given to the designer. The pedal geometry is defined with the full space of the design spaces. The brake pedal length, height, and width are approximately defined $300 \mathrm{~mm}, 30 \mathrm{~mm}$, and $18 \mathrm{~mm}$ respectively. The brake pedal is designed in SolidWorks ${ }^{\circledR}$ Software according to the dimensions that is given above. The rough design of the brake pedal is seen in Fig. 1.

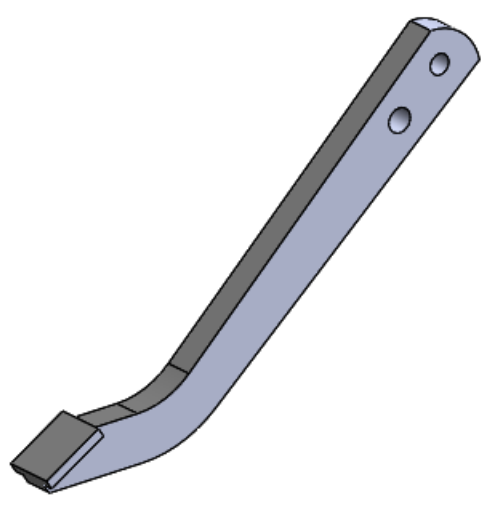

Fig. 1. Current design of brake pedal

Finite element analysis is conducted to evaluate the durability of the current brake pedal under the maximum load conditions. ANSYS ${ }^{\circledR}$ Workbench static structural module is used for the finite element analysis. The CAD data of the current brake pedal is imported ANSYS ${ }^{\circledR}$ Workbench software for the finite element analysis.

The definition of the material properties is the first step of the FE analysis. Linear static analysis is conducted in this study. Thus, the linear region of the material properties is adequate for this study. In this type, finite element analysis modulus of elasticity and Poisson ratio data is defined in the engineering data section. The material of the brake pedal is defined as S235 (Structural steel).

Table 1. Material properties of brake pedal.

\begin{tabular}{c|c}
\hline Young's modulus $(\mathrm{MPa})$ & $200,000-210,000$ \\
\hline Poisson's ratio & 0.3 \\
\hline Yield Strength $(\mathrm{MPa})$ & 235 \\
\hline Tensile Strength $(\mathrm{MPa})$ & 370 \\
\hline
\end{tabular}

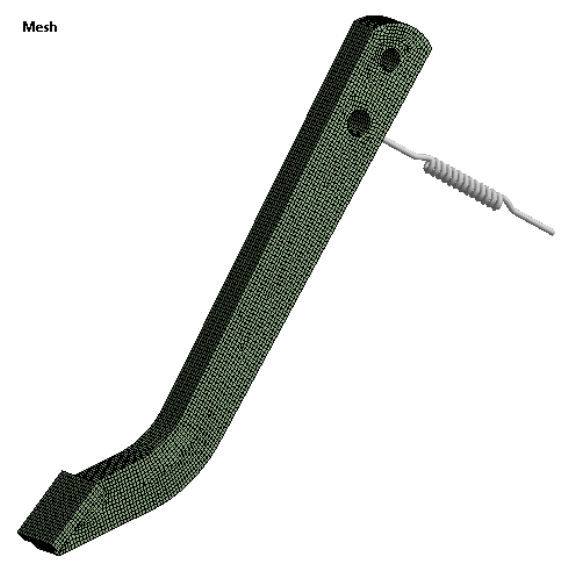

Fig. 2. Finite element mesh structure of the brake pedal 
The second phase of the finite element analysis is the definition of the mesh structure. The mesh size has great importance for the FE analysis, so the grid size should be defined carefully. If the mesh size is defined too large, the results could be untrustworthy. On the other hand, if the mesh size is defined too small, the results cloud be true, but the running time and file size increase extremely. Before the definition of the mesh size, mesh converge study is performed in this study. At first large mesh size is applied on the model, and the stress results are recorded, and the mesh size is decreased step by step when the variation of the stress results is in the tolerable level the latest mesh size is defined for the model. According to the mesh converge study, the mesh size is defined as $2 \mathrm{~mm}$; under this value, the results of the stress distribution on the brake pedal is the same. The mesh structure of the model consists of approximately 30000 hexahedral elements and 120000 nodes. A linear longitudinal spring element (COMBIN 39) is used for the definition of the hydraulic resistance force. The spring rigidity is defined as 350 $\mathrm{N} / \mathrm{mm}$ according to the hydraulic force. The finite element mesh structure is given in Fig. 2.

Cylindrical support is located at the first hole region on the brake pedal. The brake pedal turns around this hole. Thus, the only tangent rotational ability is released. Other degree of freedom is restricted. $500 \mathrm{~N}$ compression pedal force, which is applied by the driver for the maximum working conditions of the brake pedal, is added to the model. The whole boundary conditions of the static structural model are given in Fig. 3.

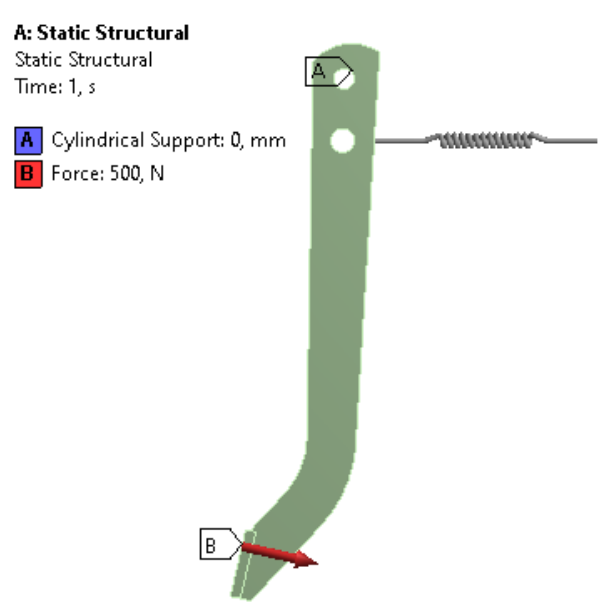

Fig. 3. Boundary conditions of finite element model

The finite element model is run after the definition of the boundary conditions. The finite element analysis type is determined as the linear static analysis in ANSYS ${ }^{\circledR}$. In static structural analysis, applied loads are not affected by time. During the analysis, whole loads, forces, moments, etc. are taken constantly. The fundamental equations of the static structural analysis are given below.

$$
\begin{aligned}
& \sum \text { Force }=\left(F_{x}, F_{y}, F_{z}\right)=0 \\
& \sum \text { Moments }=\left(M_{x}, M_{y}, M_{z}\right)=0
\end{aligned}
$$

The finite element model fulfills this condition at every node. The whole model summation of the external forces and moments is equal to the reaction forces and moments. The basic finite element equation to be solved for static loads can be expressed as:

$$
\{F\}=[K]\{u\}
$$

where $F$ is applied load vector, $K$ is the stiffness matrix, and $u$ is the displacement vector. The basic equation static structural analysis is solved by ANSYS ${ }^{\circledR}$ direct solver, and the strains are calculated. Hooke's law is used to calculate the stress distribution of the finite element model. Hooke's law is written in the following equation.

$$
[\sigma]=[E][\varepsilon]
$$

where $\sigma$ is stress, $E$ is the modulus of elasticity, and $\varepsilon$ is calculated strains.

In this study von - Mises stress is used for the equivalent stress. Equivalent stress is related to the three principal stresses at any point. It is used in maximum distortion energy theory. Equivalent stress (von - Mises) is given by:

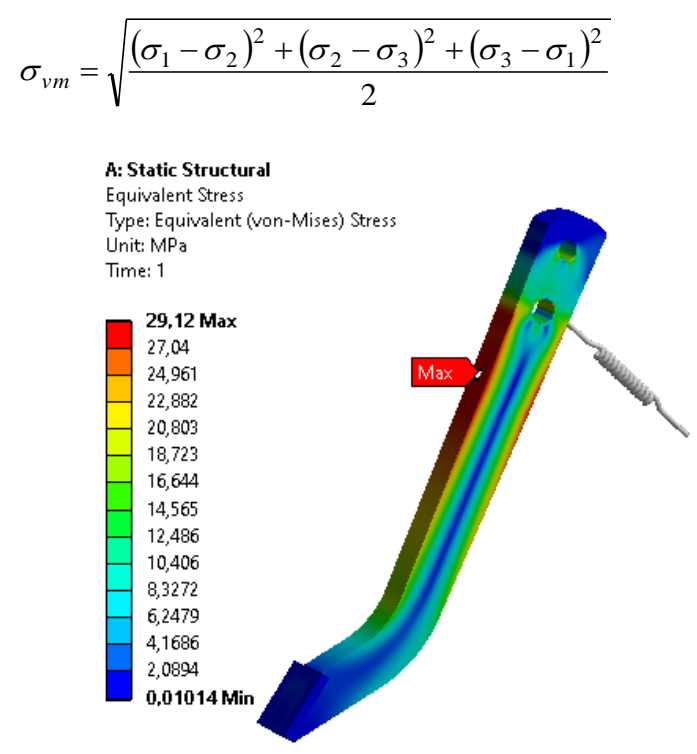

Fig. 4. Stress distribution of the current brake pedal

The stress distribution of the current brake pedal is seen in Fig. 4. It is seen that the maximum equivalent von - Mises stress is 29.12 at the top surface of the pedal. To understand whether the brake pedal acceptable or not, the designers should compare the results with particular failure criteria. 
The failure, which is used in this study, criteria are given in the following equation.

$$
\sigma_{M A X v m} \leq \sigma_{y}
$$

where $\sigma_{M A X v m}$ is maximum von - Mises stress, which is calculated by finite element analysis, and $\sigma_{y}$ yield stress of the material. When these stress values are compared, the brake pedal is safe. However, it is seen that the brake pedal is much more durable than our needs. To see this phenomenon, the safety factor for the finite element analysis is investigated. The safety factor of the static systems can be calculated as:

$$
S=\frac{\sigma_{y}}{\sigma_{M A X v m}}
$$

The safety factor distribution of the brake pedal is seen in Fig. 5. The average safety factor of this system is more than 10. The minimum safety factor is 8.58. This is too much for static even dynamic systems. It can be said that the first design of the brake pedal is overdesigned. Thus, this brake pedal should be optimized by using structural optimization techniques.

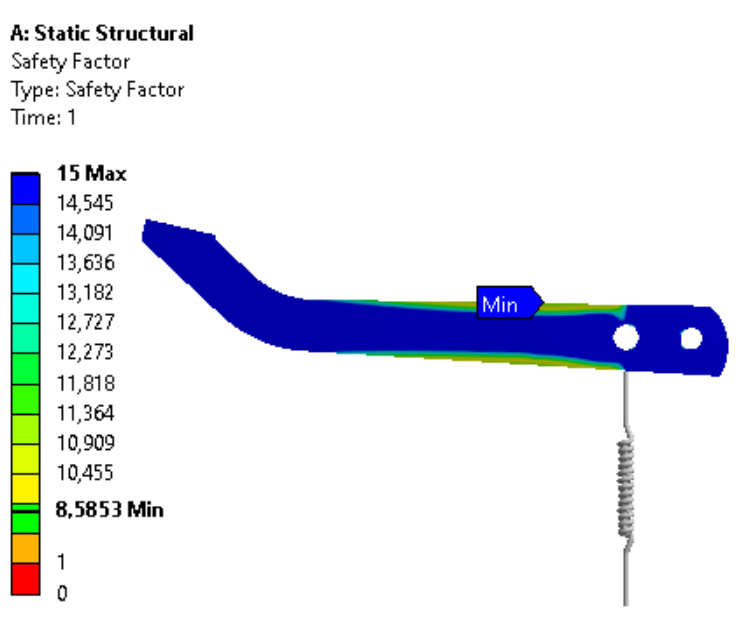

Fig. 5. Safety factor for the current brake pedal

\subsection{Topology optimization of current design}

In the general term, optimization is a process of determining the values of variables in order to maximize or minimize the objective functions under certain constraints. The objective function is the mathematical formulation of the purpose, such as maximum efficiency, minimum cost, minimum processing time, etc.

Structural optimization is a subfield of optimization which aims to find the design parameters of load-carrying mechanical structures, and it is a powerful tool in the early stages of product development. The objective function might be minimum weight, maximum strength, minimum volume, etc. while design parameters and the constraints can be selected as dimensions, mass, wall thickness, stress values, working conditions, amount of material, manufacturing constraints, etc. Structural optimization has three main methods, which are topology, shape, and size optimization.

Topology optimization seeks the optimal material layout within the predefined design space, which is given to designers, by taking into account the loads, boundary conditions, and constraints. Shape optimization, on the other hand, finds the optimum shape in predefined shape configurations which minimize an objective function while satisfying the constraints. These configurations can be determined with the help of the topology optimization and design of experiment methods. These optimization techniques are powerful tools to obtain optimum part designs.

The definition of the optimization design space is the first step of topology optimization. The whole body of the first design of the brake pedal is defined as the design surface. However, holes, the bottom surface linked with the spring, and the surface where the driver applied the load are described as non - design space for the topology optimization. The design and non - design spaces of the brake pedal is seen in Fig. 6.

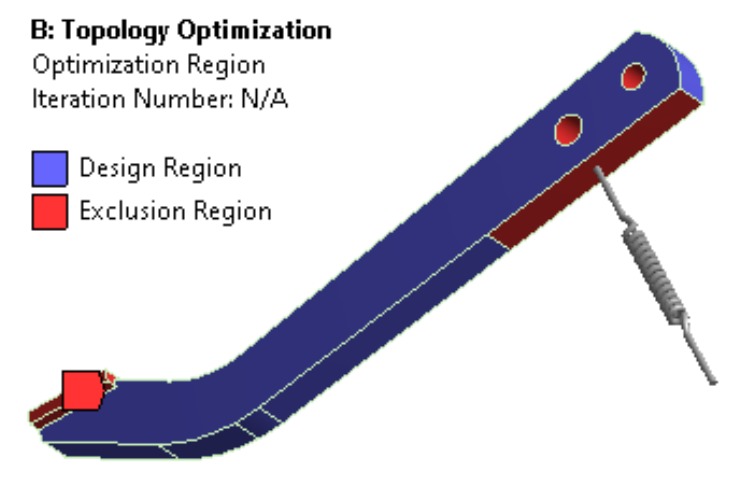

Fig. 6. Design and non - design spaces of the topology optimization

The objective is defined as the maximization of the stiffness. The topology density is defined as $50 \%$ as a constraint function, and the optimization is run. The topology optimization analysis is performed in the ANSYS ${ }^{\circledR}$ Workbench optimization module. Approximately, at the end of the thirty iterations, the optimization algorithm stopped, and the results are gained. 


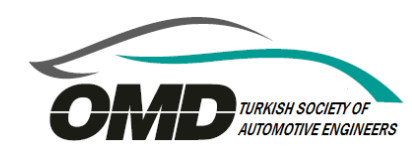

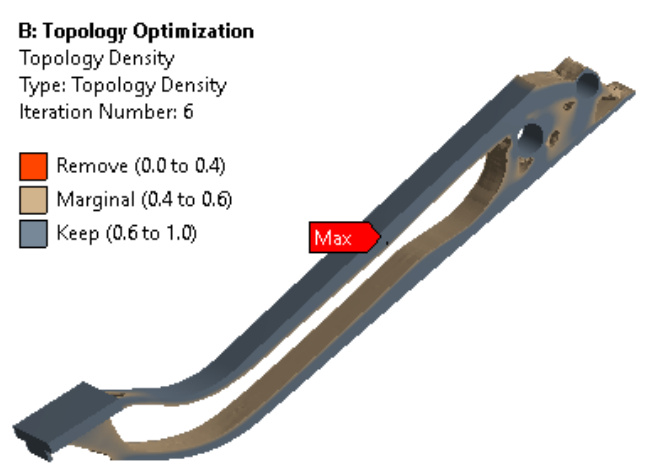

Fig. 7. Topology optimization result of brake pedal

After the topology optimization, the approximate material distribution of the brake pedal is seen in Fig. 7. According to the topology optimization results, the inner part of the brake pedal, where the stress results are minimum (Fig. 5), removed. Moreover, top region of the brake pedal is removed.

According to the topology optimization results, the brake pedal is redesigned. The inner part of the brake pedal is also removed the top region of the brake pedal is removed slightly. However, the inner part is not removed fully. Because, if the inner part of the body completely removes, the rigidity of the brake pedal sharply decreases also, the manufacturability is also decreased. It can be said that the topology optimization results are not used directly. The designer takes inspiration from the topology optimization result and creates a new design. The new design of the brake pedal is seen in Fig. 8.

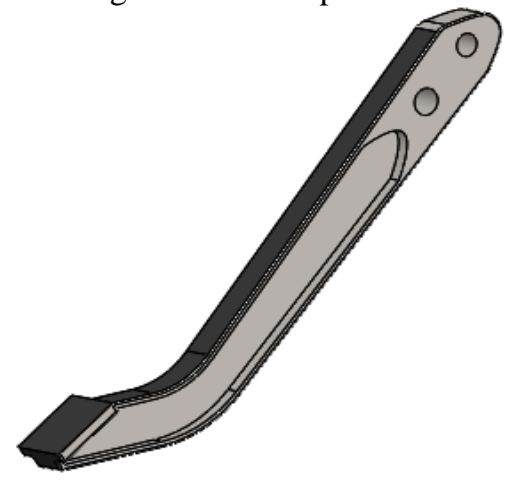

Fig. 8. New design for the brake pedal

\subsection{Design of experiment study and shape optimization}

This study used the "fmincon" function, a library function of MATLAB ${ }^{\circledR}$, to obtain an optimal design of a truck brake pedal. Thus, the optimization problem can be solved under nonlinear objective function and linear or nonlinear constraints. The "fmincon" finds a constrained minimum of a scalar function of several variables starting at an initial estimate. This is generally referred to as constrained nonlinear optimization or nonlinear programming.

Shape optimization is a process that is used for the defini- tion of the precise dimensions of the products. After the topology optimization, a new design brake pedal is gained. However, the designer does not know whether the dimensions of the new design are optimum or not. To clarify this situation, shape optimization should be done.

At the beginning of the shape optimization study, the design parameters, which will be optimized, must be defined. In this study, the wall thickness and the hole inner thickness, which are seen in Fig. 9, are taken into consideration as shape optimization design parameters.
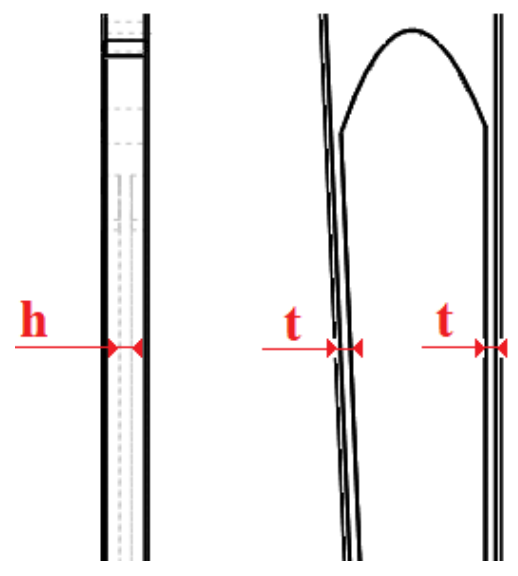

Fig. 9. Shape optimization variables

A design of experiment study is done to see the effects of these optimization parameters on the maximum equivalent von - Mises stress and the total mass of the brake pedal. Three different levels are defined both wall thickness and the hole inner thickness. A full factorial design table is created for nine different cases. In ANSYS ${ }^{\circledR}$ Workbench Design Modular, these dimensions are defined as a design parameter, and the algorithm automatically creates the model and applies the finite element analysis procedure on the model, which is described above. As a result of the design of the experiment study, the maximum equivalent von - Mises stress values are gained as seen in Table 2.

Table 2. Design of experiment table.

\begin{tabular}{c|c|c|c|c}
\hline $\begin{array}{c}\text { Experiment } \\
\text { number }\end{array}$ & $\begin{array}{c}\text { Wall } \\
\text { thickness } \\
(t)(\mathrm{mm})\end{array}$ & $\begin{array}{c}\text { Hole inner } \\
\text { thickness } \\
(h)(\mathrm{mm})\end{array}$ & $\begin{array}{c}\text { Max. Eq. } \\
\text { stress } \\
(\mathrm{MPa})\end{array}$ & Mass (g) \\
\hline 1 & 2 & 2 & 81 & 730 \\
\hline 2 & 2 & 5 & 71 & 869 \\
\hline 3 & 2 & 10 & 54 & 1099 \\
\hline 4 & 4 & 2 & 49 & 838 \\
\hline 5 & 4 & 5 & 48 & 972 \\
\hline 6 & 4 & 10 & 39 & 1163 \\
\hline 7 & 6 & 2 & 38 & 982 \\
\hline 8 & 6 & 5 & 35 & 1073 \\
\hline 9 & 6 & 10 & 33 & 1225 \\
\hline
\end{tabular}

To complete shape optimization procedure, these data 
should be express as an equation form. Different equation models are tried to find best fitted with the design of the experimental study. As a result of this study, the following equation models are used for both stress and mass equation. $\underset{H h+J}{\operatorname{Model}_{(\text {Stress })}}=A t^{2}+B t^{2} h^{2}+C t^{2} h+D t+E t h+F h^{2}+G t h^{2}+$

$\operatorname{Model}_{(\text {Mass })}=A t^{2}+B t^{2} h^{2}+C t^{2} h+D t+E t h+F h^{2}+G t h^{2}+$ $H h+J$

The coefficient of the models should be defined. The unknown coefficients of the modes were defined by using MATLAB $^{\circledR}$ surface fitting tool. When defined coefficients are written in the equations,

The equation of maximum von - Mises stress:

$$
\begin{aligned}
& F(t, h)_{\text {Stress }}=9.97917 t^{2}+0.214583 t^{2} h^{2} \\
& -3.16875 t^{2} h-8.70833 t+21.2625 t h \\
& +2.53333 h^{2}-1.70417 t h^{2}+16.6 h \\
& +614.667
\end{aligned}
$$

The equation of total mass:

$$
\begin{aligned}
& F(t, h)_{\text {Mass }}=3.71875 t^{2}+0.046875 t^{2} h^{2} \\
& -0.703125 t^{2} h-41.2708 t+6.10625 t h \\
& +0.591667 h^{2}-0.360417 t h^{2}-13.475 h \\
& +156.583
\end{aligned}
$$

The coefficient of determination of these equations is calculated at $r^{2}=0.999$. This means that the models cover well the design of the experiment table. Hence, these equations can be used in the shape optimization algorithm safety.

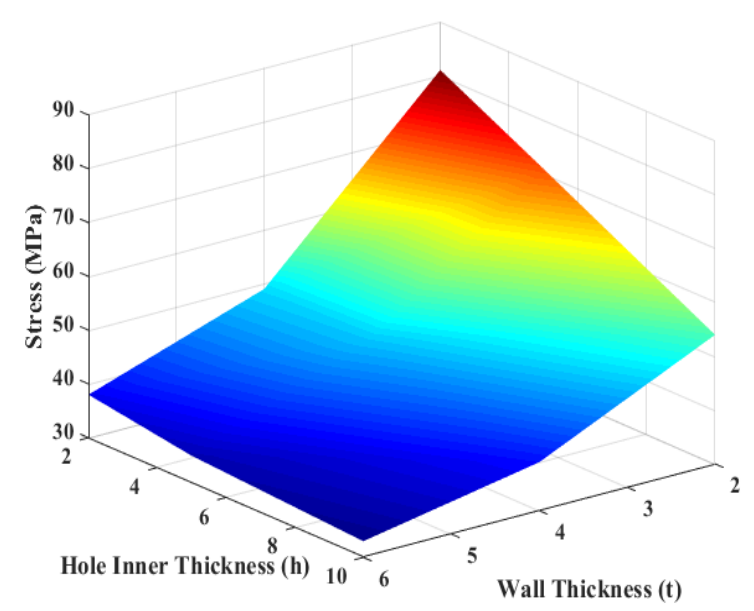

Fig. 10. Response surface of the max. von - Mises stress
11 for maximum von - Mises stress and the brake pedal total mass. The effects of hole inner thickness and wall thickness effects on the maximum von - Mises stress and the brake pedal total mass are seen easily. The effects of wall thickness on the maximum von - Mises stress is greater than the hole inner thickness. The hole inner thickness effects are also increased when the wall thickness decrease. On the other hand, the effects of hole inner thickness on the total mass are more than the wall thickness. Thus, it is not easy to find the optimum point for this design. An optimization study should be conducted to find the optimum point.

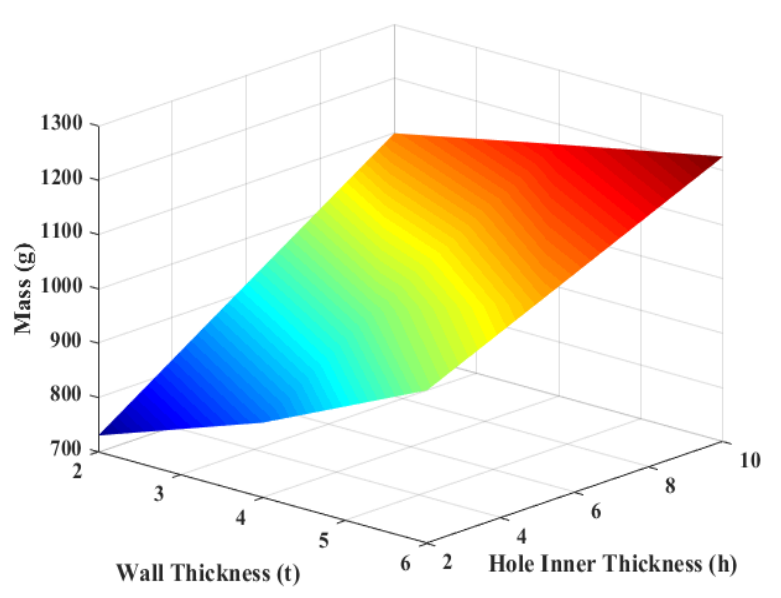

Fig. 11. Response surface of the mass

Finally, the optimization problem can be described as;

- The objective;

$$
\text { Minimize }\left[F(t, h)_{\text {Mass }}\right]
$$

- Constraints;

$$
\begin{aligned}
& F(t, h)_{\text {Stress }} \leq 75 \mathrm{MPa} \\
& 2 \leq t \leq 6 \\
& 2 \leq h \leq 10
\end{aligned}
$$

The objective function is defined as the minimum mass because the brake pedal is overdesigned, and our aim is a lightweight design. The maximum von - Mises stress is limited with the $75 \mathrm{MPa}$. Because the safety factor does not acceptable fewer than 3 for these type concept designs.

After the determination of the shape optimization problem, the problem is solved by MATLAB ${ }^{\circledR}$ "fmincon" function. To do these three different which are, objective, constraint, and main created. The main file is run, and the optimization problem is solved by MATLAB ${ }^{\circledR}$. The optimization results are given in Table 3 . 
Table 3. Shape optimization results.

\begin{tabular}{c|c}
\hline Stress $(\mathrm{MPa})$ & 75 \\
\hline Mass $(\mathrm{g})$ & 744 \\
\hline Wall thickness $(t)(\mathrm{mm})$ & 2.3 \\
\hline Hole inner thickness $(h)(\mathrm{mm})$ & 2 \\
\hline
\end{tabular}

According to the optimization results, hole inner thickness is defined as $2 \mathrm{~mm}$, and the wall thickness is defined as 2.3 $\mathrm{mm}$. The maximum von - Mises equivalent stress is $75 \mathrm{MPa}$, which is the top acceptable stress value, and the mass of the brake pedal is calculated 744 grams according to the new dimension of the brake pedal.

After the shape optimization study, the new optimized design is seen in Fig. 12.

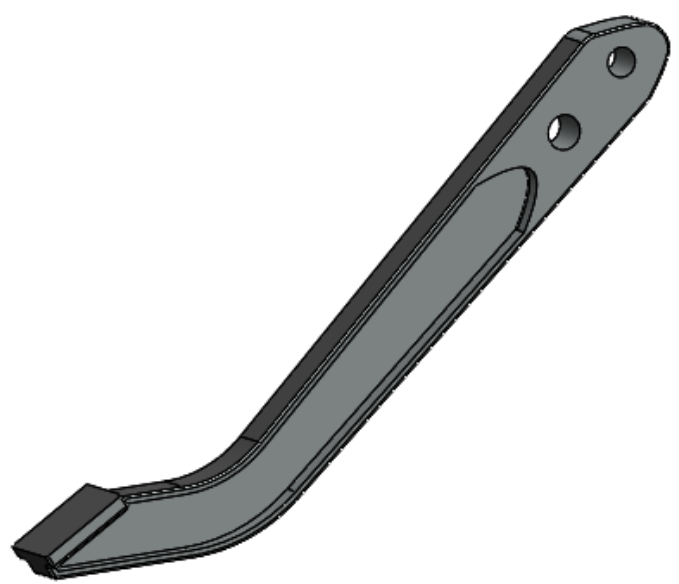

Fig. 12. New brake pedal design after shape optimization

A final finite element analysis is done to verify the new optimized design of the brake pedal. The finite element analysis, which is explained in section 2.1, repeated with the optimized model of brake pedal. All mesh structure and the boundary conditions are taken the same as the first analysis.

As a result of the finite element analysis, the maximum equivalent von - Mises stress values are calculated as 80 MPa. After the shape optimization study, this value is calculated as $75 \mathrm{MPa}$. The differences between shape optimization and the finite element analysis are only $5 \mathrm{MPa}$, so this difference is acceptable.

Table 4. Comparison of current and optimized brake pedal.

\begin{tabular}{c|c|c|c}
\hline & $\begin{array}{c}\text { Current brake } \\
\text { pedal }\end{array}$ & $\begin{array}{c}\text { Optimized } \\
\text { brake pedal }\end{array}$ & Result \\
\hline Stress $(\mathrm{MPa})$ & 29.12 & 80.01 & Acceptable \\
\hline Mass $(\mathrm{g})$ & 1500 & 744 & $-50.04 \%$ \\
\hline
\end{tabular}

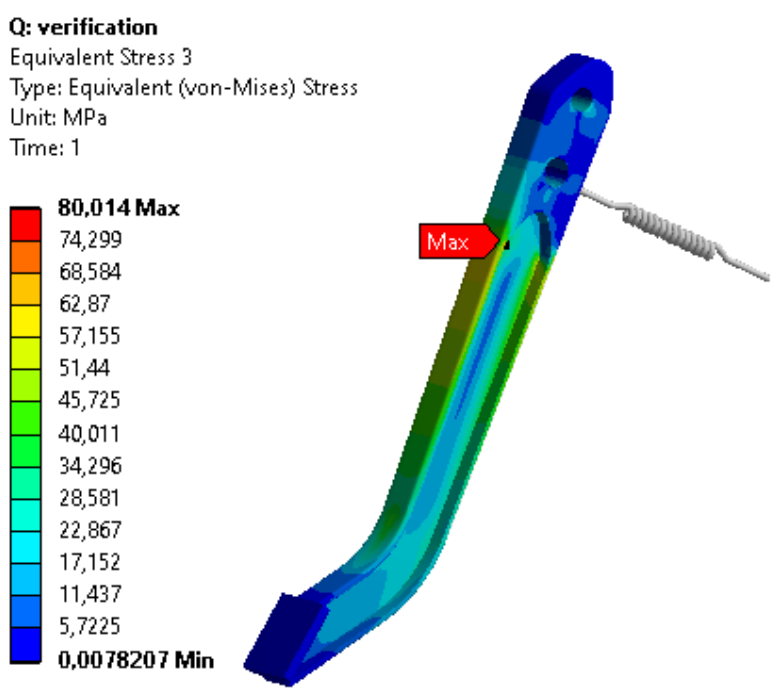

Fig. 13. Design verification of the new brake pedal

The comparison of first and optimized designs is given in Table 4. According to the results, the overdesigned brake pedal is optimized. The stress values are increasing. However, the increase in the stress values is in the acceptable borders. On the other hand, the mass of the brake pedal is nearly decreased by $50 \%$. By using the structural optimization techniques, design of experiment study, and finite element method, a design procedure for a brake pedal is created.

\section{Conclusions}

Because of its positive impacts on vehicles and environments such as low manufacturing cost, emission, and fuel consumption reduction, etc., lightweight vehicle designs have become one of the important subjects of the automotive industry.

In this study, structural optimization and design of experiment methods were used in order to obtain an optimum and also lighter design of a heavy truck brake pedal. After finite element analysis was conducted for rough design of the brake pedal, it has seen that pedal was overdesigned according to Fig. 5. Therefore, topology optimization was performed to obtain optimum material layout (Fig. 7), and the brake pedal is redesigned considering optimization results and other factors such as rigidity and manufacturability. In the new design, wall thickness and hole inner thickness were taken as design parameters. Then, the design of the experimental method and shape optimization was used respectively to find the optimum dimensions of these design parameters. Results were validated by performing finite element analysis for the new design of the brake pedal. 
As a result of these studies, design parameters, wall thickness, and hole inner thickness were calculated 2.3 and $2 \mathrm{~mm}$, and with these values, approximately $\% 50$ lighter design of a brake pedal of the heavy truck has been achieved. Although maximum stress value is increased up to $80 \mathrm{MPa}$, it is within the acceptable stress limit. Therefore, this study indicates that the structural optimization and design of experiment methods are powerful tools to design lighter vehicle parts in the early stages of product development.

\section{References}

[1] Dogan, O., Karpat, F., Yuce, C., Kaya, N., Yavuz, N. and Sen, H. (2016). A novel design procedure for tractor clutch fingers by using optimization and surface response methods. Journal of Mechanical Science and Technology, 30, 2615-2625.

[2] Karpat, F., Doğan, O. and Yüce, C. (2017). Shape optimization of an overdesigned chain link by using design of experiment graphical optimization. Proceeding of Academic World $66^{\text {th }}$ International Conference, 21-22 May, Prague, Czech Republic.

[3] Dogan, O., Karpat, F., Kaya, N., Yuce, C., Genc, M. O. and Yavuz, N. (2015). Optimum design of tractor clutch PTO finger by using topology and shape optimization. Proceedings of the ASME 2015 International Mechanical Engineering Congress and Exposition, November 13-19, Houston, Texas.

[4] Ergenc, A. F., Ergenc, A. T., Kale, S., Sahin, I. G., Pestelli, V., Dagdelen, K., Yontem, O. and Kuday, B. (2017). Reduced weight automotive brake pedal test \& analysis. International Journal of Automotive Science and Technology, 1, 8-13.

[5] Kaya, N., Karen, İ. and Öztürk, F. (2010). Redesign of a failed clutch fork using topology and shape optimization by the response surface method. Materials and Design, 31, 3008-3014.

[6] Cavazzuti, M., Baldini, A., Bertocchi, E., Costi, D., Torricelli, E. and Moruzzi, P. (2011). High performance automotive chassis design: a topology optimization based approach. Structural and Multidisciplinary Optimization, 44, 45-56.

[7] Kurkure, B. and Sadaphale, D. B. (2015). Optimization of brake pedal. International Journal of Mechanical and Industrial Technology, 3, 175-183.

[8] Ebrahimi, M. and Behdinan, K. (2015). A novel approach for design and optimization of automotive aluminum cross-car beam assemblies. SAE Technical Paper (No. 2015-01-1370).

[9] Chang, J. W. and Lee, Y. S. (2008). Topology optimization of compressor bracket. Journal of Mechanical Science and Technology, 22, 1668-1676.

[10]Sudin, M. N., Tahir, M. M., Ramli, F. R. and Shamsuddin, S. A. (2014). Topology optimization in automotive brake pedal redesign. International Journal of Engineering and Technology, 6, 398-402.

[11]Dhande, K.K., Jamadar, N.I. and Ghatge, S. (2014). Conceptual design and analysis of brake pedal profile. International Journal of Innovative Research in Science, Engineering and Technology, 3, 17432-17441.
[12]Romero, J. and Queipo, N. (2017). Reliability-based and deterministic design optimization of a FSAE brake pedal: a risk allocation analysis. Structural and Multidisciplinary Optimization, 56, 681-695. 